EPJ Web of Conferences 64, 04005 (2014)

DOI: $10.1051 /$ epjconf/ 20146404005

(C) Owned by the authors, published by EDP Sciences, 2014

\title{
3D Gray Radiative Properties of Accretion Shocks in Young Stellar Objects
}

\author{
L. Ibgui ${ }^{1, a}$, S. Orlando ${ }^{2, b}$, C. Stehlé ${ }^{1, c}$, J.-P. Chièze ${ }^{3, d}$, I. Hubeny ${ }^{4, e}$, T. Lanz ${ }^{5, f}$, L. de Sá ${ }^{1,3, g}$, \\ T. Matsakos ${ }^{1,3,6, h}$, M. González ${ }^{3, i}$, and R. Bonito ${ }^{2,7, j}$ \\ ${ }^{1}$ LERMA, Observatoire de Paris, CNRS et UPMC, 5 Place J. Janssen, 92195 Meudon, France \\ ${ }^{2}$ INAF - Osservatorio Astronomico di Palermo, Piazza del Parlamento, 1 , 90134, Palermo, Italy \\ ${ }^{3}$ Laboratoire AIM, IRFU / Service d'Astrophysique - CEA/DSM - CNRS - Université Paris Diderot, Bât. 709, \\ CEA-Saclay, F-91191 \\ ${ }^{4}$ Steward Observatory, University of Arizona, 933 North Cherry Avenue, Tucson, AZ 85721, USA \\ ${ }^{5}$ Laboratoire J.-L. Lagrange, Université de Nice-Sophia Antipolis, CNRS, Observatoire de la Côte d'Azur, \\ BP 4229, 06304 Nice, France \\ ${ }^{6}$ CEA, IRAMIS, Service Photons, Atomes et Molécules, 91191 Gif-sur-Yvette, France \\ ${ }^{7}$ Dip. di Fisica e Chimica, Università degli Studi di Palermo, Piazza del Parlamento, 1, 90134, Palermo, Italy
}

\begin{abstract}
We address the problem of the contribution of radiation to the structure and dynamics of accretion shocks on Young Stellar Objects. Solving the 3D RTE (radiative transfer equation) under our "gray LTE approach", i.e., using appropriate mean opacities computed in local thermodynamic equilibrium, we post-process the 3D MHD (magnetohydrodynamic) structure of an accretion stream impacting the stellar chromosphere. We find a radiation flux of ten orders of magnitude larger than the accreting energy rate, which is due to a large overestimation of the radiative cooling. A gray LTE radiative transfer approximation is therefore not consistent with the given MHD structure of the shock. Further investigations are required to clarify the role of radiation, by relaxing both the gray and LTE approximations in RHD (radiation hydrodynamics) simulations. Post-processing the obtained structures through the resolution of the non-LTE monochromatic RTE will provide reference radiation quantities against which RHD approximate solutions will be compared.
\end{abstract}

\footnotetext{
ae-mail: laurent.ibgui@obspm.fr

be-mail: orlando@astropa.inaf.it

ce-mail: chantal.stehle@obspm.fr

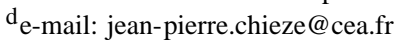

e e-mail: hubeny@as.arizona.edu

fe-mail: thierry.lanz@oca.eu

ge-mail: lionel.de-sa@cea.fr

he-mail: titos.matsakos@cea.fr

i e-mail: matthias.gonzalez@cea.fr

je-mail: sbonito@astropa.unipa.it
}

This is an Open Access article distributed under the terms of the Creative Commons Attribution License 2.0, which permits unrestricted use, distribution, and reproduction in any medium, provided the original work is properly cited. 


\section{Physics of Accretion Shocks on Young Stellar Objects}

Understanding the process of star formation is a major challenge in modern astrophysics. Among young stellar objects (YSO), the classical T Tauri stars (CTTSs) are of considerable interest, because they have a mass comparable to that of the Sun, and they accrete material from a circumstellar disk. Magnetospheric accretion is the reference scenario adopted for CTTSs [1,2]. Within a few stellar radii, the accretion disk dynamics is controlled by the stellar magnetic field (of the order of $\mathrm{kG}$ ): the ionized disk is truncated, the matter falls onto the stellar surface along magnetic funnels and impacts the stellar surface at free-fall speed, which results in the formation of radiative shocks. Several large scale magnetohydrodynamic multidimensional accretion simulations were performed in the past years $[3,4]$. However, they do not tackle the problem of the area of impact, i.e., the interaction of the accretion stream with the stellar surface, where an accretion shock is formed, which is the source of intense radiation emission. The scientific community has accumulated, for twenty years, a wealth of spectra, from medium to high resolution, that range from soft X-ray to near infrared regions [5, 6]. Theory is lagging far behind observations: providing a consistent and thorough model of accretion onto young stellar objects is a major theoretical challenge. Many open issues are being debated about the physics of accretion shocks, on the basis of observations or theoretical results, such as the stability of the shock (presence or absence of quasi-periodic oscillations), the potential existence of a precursor (radiatively heated pre-shock region), to name a few.

Current models include 1D hydrodynamic or 2D MHD simulations [7-13]. In all of these models, radiation contribution is restrained to a radiative loss term in the energy equation, assuming an optically thin plasma; there is no radiation contribution to the momentum equation. Yet, radiative transfer may be a crucial ingredient influencing the structure and dynamics of an accretion shock. Chièze et al. [14] and de Sá et al. [15] have, for the first time, proposed 1D radiation hydrodynamics simulations of an accretion shock on a young stellar object, along with acoustic heating of the chromosphere. Their preliminary results show that an efficient radiation gas coupling may suppress the oscillations induced by cooling instability. In this approach, radiative transfer is modeled with the approximate M1 method in the inner LTE regions ([16-18]) using Rosseland and Planck mean opacities, and a coronal regime in the non-LTE external region. The transition between both regimes is controlled by the escape probability of photons through the accretion column (see [15] for details).

We propose to perform the most accurate radiation calculation, by solving the radiative transfer equation (RTE), and then inferring the radiation terms in the momentum and energy equations. This way, we can assess the possibility of a radiative feedback on the dynamics of an accretion shock, by comparing the radiative moments to the thermal and magnetic quantities, as explained below.

\section{Radiative feedback on the dynamics of an accretion shock, using the resolution of the RTE}

The ideal approach of the coupling between radiation and hydrodynamics is to solve the monochromatic radiative transfer equation, in order to infer the radiation moments that are involved in the gas momentum and energy equations. It is well known that this approach is out of reach of current computer capabilities, which justifies the development of approximate radiation models (see section 1 above). Yet, it is possible to solve the monochromatic RTE for a given snapshot of hydrodynamics, RHD, or MHD simulations, and then to infer the radiation quantities that are involved in the dynamics equations (momentum and energy).

We discuss below first results of the latter approach. The structure of the accretion shock is modeled with PLUTO [19, 20], which is a MHD Godunov-type code for astrophysical plasmas. PLUTO 
includes optically thin radiative losses modeled with a cooling function $\Lambda(T)$ that depends on temperature $T$, and that is derived from the APED V1.3 atomic line database [21], assuming complete ionization of the plasma. The accretion column is modeled with a $2 \mathrm{D}$ axisymmetric simulation, from which we have built a 3D structure by rotation around the accretion direction represented by a z-axis. MHD equations and details on the basic physical modeling are described in [10]. The accretion stream is perpendicular to the chromosphere. The magnetic field is strong $(500 \mathrm{G})$, so that the plasma $\beta$ parameter $\left(\beta=p_{\text {gas }} / p_{\text {magnetic }}<1\right)$. Figure 1 shows the structure of the accreting column and its surrounding environment.

We propose to determine the radiative components of the momentum and energy conservation equations, by solving the 3D RTE with the IRIS code [22]. While approximate radiative transfer models are required to make radiation hydrodynamics calculations tractable (e.g., [15]), the RTE solution provides, for a given set of opacities, the most accurate contribution of radiation to dynamics. Because of computational challenges, such a contribution can be calculated only for one or some snapshots of hydrodynamics or MHD simulations, and cannot be directly coupled with the dynamics equations. Before embarking on the resolution of the non-LTE monochromatic RTE, which is a long journey, we have investigated gray approaches.

The first model we consider uses Rosseland and Planck mean opacities, derived from monochromatic opacities calculated with the SYNSPEC code [23] (under the assumption of LTE). We find a radiation flux in the accretion stream direction that is about ten orders of magnitude larger than the energy rate carried by the accreting column. This discrepancy stems from the fact that such a LTE treatment of radiation results in a large overestimation of the radiative cooling of the gas. Another option that we have explored is to define a gray emission coefficient, $\eta_{\Lambda}$, such as the radiative losses calculated from RTE resolution equal to the radiative losses considered in MHD simulations through the $\Lambda$ function ( $=n_{e} n_{H} \Lambda(T)$, cf.[10], where $n_{e}$ and $n_{H}$ are the electron and hydrogen densities). In this case, we have defined an absorption coefficient $\chi_{\Lambda}$ related to $\eta_{\Lambda}$ through the LTE source function: $S(T)=\sigma T^{4} / \pi=\eta_{\Lambda} / \chi_{\Lambda}$, where $\sigma$ is the Stefan-Boltzmann constant. Such a treatment leads, also, to an overestimation of about ten orders of magnitude of the radiation flux. As a matter of fact, we find a cooling time (internal energy of the gas divided by the divergence of the radiation flux) of the order of $10 \mathrm{~ns}$, which means that such a MHD structure (as calculated in the optically thin limit) is heavily incompatible with LTE gray radiation transport.

Further investigations need to be done. In particular, we plan to relax the gray approximation, by solving, still with IRIS, the monochromatic RTE, assuming LTE opacities. Then, we will adopt a nonLTE approach. In parallel to that project, we continue investigating radiation hydrodynamics models (with approximate radiation treatment), including transition between optically thick and optically thin regimes within the accretion column [15].

\section{Acknowledgements}

The work is supported by French ANR, under grant 08-BLAN-0263-07.

\section{References}

[1] M. Camenzind, in Reviews in Modern Astronomy, edited by G. Klare (1990), Vol. 3, pp. 234-265

[2] A. Königl, ApJL 370, L39 (1991)

[3] M.M. Romanova, G.V. Ustyugova, A.V. Koldoba, R.V.E. Lovelace, ApJ 610, 920 (2004)

[4] M. Long, M.M. Romanova, A.K. Kulkarni, J.F. Donati, MNRAS 413, 1061 (2011)

[5] S. Alencar, in EPJ - This conference (2013) 


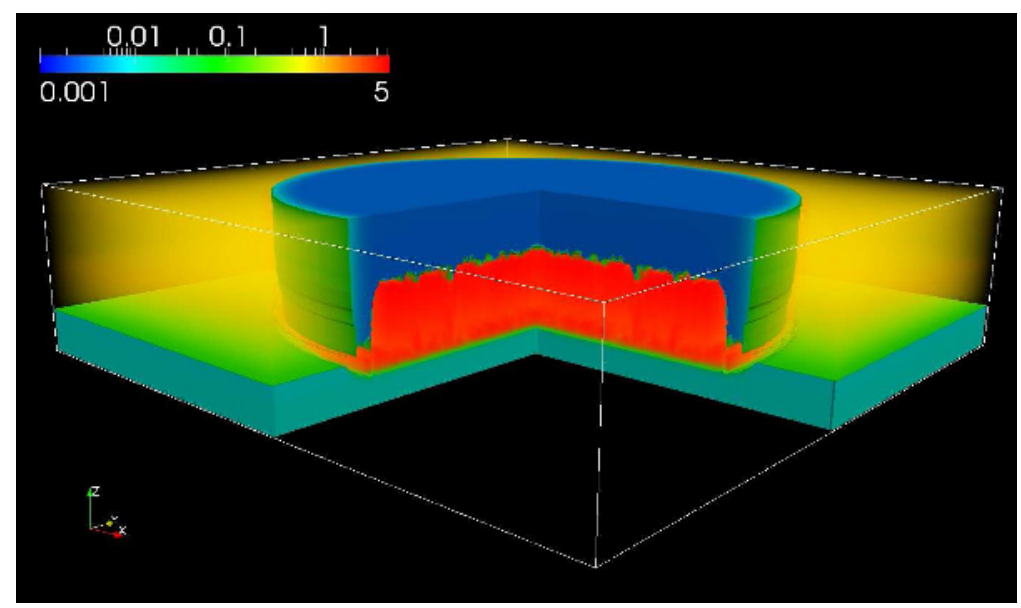

Figure 1. Temperature (MK) of an accreting structure, for a given PLUTO MHD snapshot. The corona is at $\sim 0.9 \mathrm{MK}$ (yellow). The unperturbed chromosphere is at $\sim 10^{4} \mathrm{~K}$ (light blue). The accreting column is made of the pre-shock plasma at $\sim 2500 \mathrm{~K}$ (dark blue), and the post-shock plasma at $\sim 5 \mathrm{MK}$ (red).

[6] C. Johns-Krull, in EPJ - This conference (2013)

[7] G.G. Sacco, C. Argiroffi, S. Orlando, A. Maggio, G. Peres, F. Reale, A\&A 491, L17 (2008)

[8] G.G. Sacco, S. Orlando, C. Argiroffi, A. Maggio, G. Peres, F. Reale, R.L. Curran, A\&A 522, A55 (2010)

[9] A.V. Koldoba, G.V. Ustyugova, M.M. Romanova, R.V. Lovelace, MNRAS 388, 357 (2008)

[10] S. Orlando, G.G. Sacco, C. Argiroffi, F. Reale, G. Peres, A. Maggio, A\&A 510, A71 (2010)

[11] T. Matsakos, J.P. Chièze, C. Stehlé, M. González, L. Ibgui, L. de Sá, T. Lanz, S. Orlando, R. Bonito, C. Argiroffi et al., A\&A, in press (2013)

[12] T. Matsakos, in EPJ - This conference (2013)

[13] S. Orlando, R. Bonito, C. Argiroffi, G. Reale, F. Peres, M. Miceli, T. Matsakos, C. Stehlé, L. Ibgui, L. de Sa, J.P. Chièze et al., submitted to A\&A (2013)

[14] J.P. Chièze, L. de Sá, C. Stehlé, in EAS Publications Series (2012), Vol. 58, pp. 143-147

[15] L. de Sá, J.P. Chièze, C. Stehlé, T. Matsakos, L. Ibgui, T. Lanz, I. Hubeny, in EPJ - This conference (2013)

[16] C.D. Levermore, JQSRT 31, 149 (1984)

[17] C.D. Levermore, Journal of Statistical Physics 83, 1021 (1996)

[18] B. Dubroca, J. Feugeas, CRAS 329, 915 (1999)

[19] A. Mignone, G. Bodo, S. Massaglia, T. Matsakos, O. Tesileanu, C. Zanni, A. Ferrari, ApJS 170, 228 (2007)

[20] A. Mignone, C. Zanni, P. Tzeferacos, B. van Straalen, P. Colella, G. Bodo, ApJS 198, 7 (2012)

[21] R. Smith, N. Brickhouse, D. Liedahl, J. Raymond, ApJL 556, L91 (2001)

[22] L. Ibgui, I. Hubeny, T. Lanz, C. Stehlé, A\&A 549, A126 (2013)

[23] I. Hubeny, T. Lanz, Astrophysics Source Code Library (2011) 